\title{
Diagrammatic Monte Carlo simulations of staggered fermions at finite coupling
}

\author{
Hélvio Vairinhos* \\ Institute for Theoretical Physics, ETH Zürich, CH-8093 Zürich, Switzerland \\ E-mail: helviov@ethz.phys.ch

\section{Philippe de Forcrand} \\ Institute for Theoretical Physics, ETH Zürich, CH-8093 Zürich, Switzerland \\ CERN, Physics Department, TH Unit, CH-1211 Geneva 23, Switzerland \\ E-mail: forcrandethz.phys.ch
}

\begin{abstract}
Diagrammatic Monte Carlo has been a very fruitful tool for taming, and in some cases even solving, the sign problem in several lattice models. We have recently proposed a diagrammatic model for simulating lattice gauge theories with staggered fermions at arbitrary coupling, which extends earlier successful efforts to simulate lattice QCD at finite baryon density in the strong-coupling regime. Here we present the first numerical simulations of our model, using worm algorithms.
\end{abstract}

The 33rd International Symposium on Lattice Field Theory

14 - 18 July 2015

Kobe International Conference Center, Kobe, Japan*

\footnotetext{
${ }^{*}$ Speaker.
} 


\section{Introduction}

The numerical simulation, from first principles, of lattice QCD at finite density is an amazingly hard task to perform, due to the notorious sign problem [1]. Traditionally, the Grassmann variables in the partition function of lattice QCD are integrated analytically a priori, and Monte Carlo simulations consist of sampling the gauge field $U$, weighted by the pure gauge action $S_{g}(U)$, and by the Dirac determinant which results from such an integration:

$$
Z=\int[\mathscr{D} \chi \mathscr{D} \bar{\chi} \mathscr{D} U] e^{-S_{g}(U)+\bar{\chi} \not D(\mu, m) \chi}=\int[\mathscr{D} U] e^{-S_{g}(U)} \operatorname{det}(\not D(\mu, m))
$$

However, such a determinant is complex-valued for any non-zero real chemical potential. This undermines the probabilistic interpretation of the Boltzmann weight, and prevents direct numerical simulations.

The sign problem is, however, representation-dependent, in the sense that it depends on the basis of the Hilbert space over which one decides to trace, in order to obtain the partition function, $Z=\operatorname{Tr}\left(e^{\beta \hat{H}}\right)$. In the energy eigenbasis $\left|\Psi_{i}\right\rangle$, for example, the sign problem is absent by definition, since every element of the transfer matrix is real non-negative: $\left\langle\Psi_{i}\left|e^{-\beta \hat{H} \delta t}\right| \Psi_{j}\right\rangle \geq 0, \forall i, j$. A solution to the sign problem in lattice QCD thus amounts to finding an adequate basis of states with respect to which the sign problem is at least mild and tractable (e.g. with reweighing methods).

Such a basis exists in the strong coupling limit of lattice QCD: it consists of constrained worldlines of color-neutral states (mesonic monomers and dimers, and baryonic loops), which result from integrating out the link variables before the Grassmann variables [2]. This representation of the partition function is purely diagrammatic, and efficient worm-like algorithms exist which simulate it efficiently [3]. The sign problem is so mild that it allows to map the full phase diagram of strong coupling lattice $\mathrm{QCD}$, in the strong coupling limit and at $O(\beta)$ [4].

The reason for this success is that the relevant group integrals in the strong coupling limit and at $O(\beta)$ are simple enough, so that exact diagrammatic representations of the partition function can be found. At higher orders in $\beta$, however, the task of computing the relevant group integrals becomes cumbersome, and a different approach is desirable to tackle the problem at arbitrary $\beta$.

\section{Compact lattice QED without link variables}

Group integrals in lattice gauge theory are difficult to solve for arbitrary $\beta$ because of the terms in the Wilson gauge action, which couple four links around a plaquette. In the strong coupling limit $(\beta=0)$, the plaquette terms vanish, and the partition function factorizes as a product of one-link integrals, whose exact solution is known for many gauge groups.

In order for exact integration to be possible for any $\beta$, we introduce a set of free auxiliary Gaussian variables on each plaquette (see Fig.1), and couple them to the link variables via HubbardStratonovich (HS) transformations [5]. The auxiliary variables can be made to couple to a single link variable, while canceling higher-coupling terms (including all the Wilson plaquette terms). The partition function thus reduces to a Gaussian integral over the auxiliary variables, whose integrand factorizes as a product of solvable one-link integrals [5]. This method can be easily generalized in the presence of any number of staggered flavours, coupled to a chemical potential or not. 


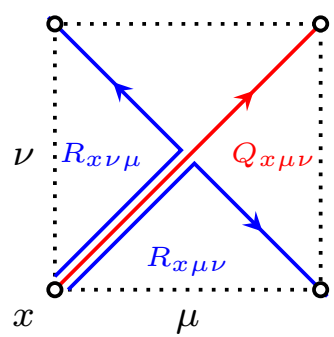

Figure 1: Auxiliary variables which decouple plaquette terms into sums of one-link terms.

Here we exemplify with the case of a $U(1)$ gauge group and $N_{f}=1$, i.e. compact lattice QED with one staggered fermion. The fermion does not couple to a chemical potential due to Gauss' law. However, it serves as a good toy model with which we can test diagrammatic Monte Carlo methods at arbitrary values of $\beta$.

When the method of auxiliary variables [5] is applied to the partition function of $N_{f}=1$ compact lattice QED, it becomes a weighted sum over lattice diagrams:

$$
\begin{aligned}
Z & =\int[d U] \prod_{p} e^{\beta \operatorname{Re}\left(U_{p}\right)} \prod_{x, \mu} e^{\xi_{\mu} \bar{\chi}_{x} U_{x \mu} \chi_{x+\hat{\mu}}-\xi_{\mu} \bar{\chi}_{x+\mu} U_{x \mu}^{\dagger} \chi_{x}} \prod_{x} e^{2 a m \bar{\chi}_{x} \chi_{x}} \\
& =\int \mathscr{G}_{\beta}[Q, R] \prod_{x} I_{0}\left(\beta\left|J_{x \mu}\right|\right) \sum_{\{n, k, C\}} \sigma_{F}(C) \prod_{x}(2 a m)^{n_{x}} \prod_{x, \mu} \xi_{\mu}^{2 k_{x \mu}} \prod_{i=1}^{\# C} 2 \operatorname{Re}\left(\mathscr{U}\left(C_{i}\right)\right)
\end{aligned}
$$

where $a m$ is the bare mass of the staggered fermion in lattice units; $\xi$ is the anisotropy parameter; $\mathscr{G}_{\beta}[Q, R]=[\mathscr{D} Q \mathscr{D} R] e^{-\frac{3 \beta}{2} Q Q^{\dagger}} e^{-\frac{\beta}{2} R R^{\dagger}}$ is the product of Gaussian measures of all auxiliary variables; $I_{0}$ is a modified Bessel function of the first kind, which is the solution of the $U(1)$ one-link integral:

$$
\int d U e^{\beta \operatorname{Re}\left(J_{x \mu}^{\dagger} U\right)}=I_{0}\left(\beta\left|J_{x \mu}\right|\right),
$$

whose argument involves the effective staples:

$$
J_{x \mu}=\sum_{v \neq \mu} \frac{\xi_{\mu v}}{\xi^{2}}\left(R_{x-\hat{v}, v \mu}^{\dagger} Q_{x-\hat{v}, v \mu}+R_{x \mu v}\right)
$$

$\{n, k, C\}$ represents a configuration of lattice diagrams, in which $n_{x} \in\{0,1\}$ is the occupation number of monomers, $k_{x \mu} \in\{0,1\}$ is the occupation number of dimers, and $C$ is a disjoint union of self-avoiding fermionic loops $C_{i}$, with \#C connected components. These diagrams are constrained by Grassmann integration in that only one fermion and one anti-fermion per site are allowed (see Fig.2); $\sigma_{F}(C)= \pm 1$ is a sign which depends on the geometry and topology of each connected fermion loop $C_{i} ; \mathscr{U}\left(C_{i}\right)$ is the product of effective links $\mathscr{U}_{l}$ around $C_{i}$, and an effective link is the expectation value of the link variable with respect to the one-link integral:

$$
\mathscr{U}_{l}=\langle U\rangle_{J_{l}}=\int d U U e^{\beta \operatorname{Re}\left(J_{l}^{\dagger} U\right)}=\frac{I_{1}\left(\beta\left|J_{l}\right|\right)}{I_{0}\left(\beta\left|J_{l}\right|\right)} \frac{J_{l}}{\left|J_{l}\right|}
$$

\section{Monte Carlo simulation}

The partition function of lattice QED in the diagrammatic form Eq.(2.2) is amenable to diagrammatic Monte Carlo methods. In particular, a generalization of the worm-inspired directed- 


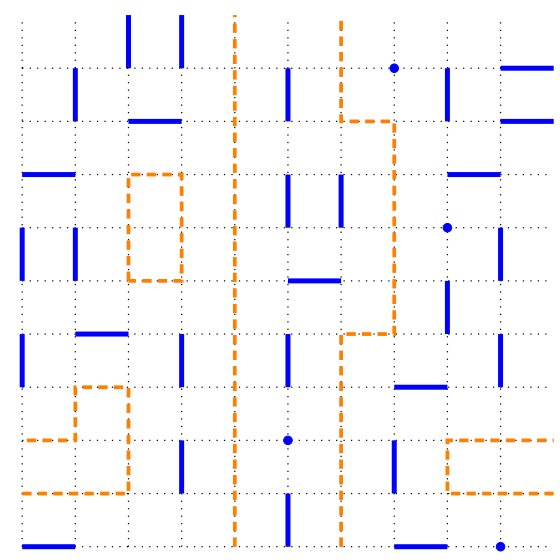

Figure 2: Admissible configuration of the lattice massive Schwinger model in the diagrammatic representation at finite $\beta$.

path algorithm (dpa) for the strong coupling limit of $S U(N)$ lattice gauge theories with staggered fermions [3] can be used to sample efficiently the fermionic worldlines: the monomer-dimer sector is updated using a mesonic worm, i.e. a worm which carries a monomer defect on its tail, while the dimer-loop sector is updated using a fermionic worm, i.e. a worm which carries a single fermion defect on its tail. The setup is similar to the dpa used in [4], but generalized to the situation in which some links (the ones touched by fermion loops) carry an extra weight depending on the magnitude of the corresponding effective link.

Auxiliary variables, on the other hand, are updated with a Gaussian heatbath, plus a Metropolis update which corrects the distribution of auxiliary variables for the presence of fermion loops.

In order to validate our code, we test it on small lattices for which we can compute the partition function analytically. In order to test the mesonic worm algorithm, which leaves the fermionic loops invariant, we simulate compact lattice QED on a $2 d$ lattice (massive Schwinger model), at $\beta=0$. The partition function in this case can be computed analytically for small enough lattices as a function of the bare fermion mass and lattice anisotropy; for example, on a $4 \times 4$ lattice the partition function is given by:

$$
\begin{aligned}
Z(\xi, m)= & 16\left(\xi^{16}+4 \xi^{12}+7 \xi^{8}+4 \xi^{4}+1\right)+(2 a m)^{16}+16\left(\xi^{2}+1\right)(2 a m)^{14} \\
& +8\left(13 \xi^{4}+24 \xi^{2}+13\right)(2 a m)^{12}+32\left(\xi^{2}+1\right)\left(11 \xi^{4}+17 \xi^{2}+11\right)(2 a m)^{10} \\
& +8\left(83 \xi^{8}+256 \xi^{6}+354 \xi^{4}+256 \xi^{2}+83\right)(2 a m)^{8} \\
& +64\left(11 \xi^{10}+37 \xi^{8}+63 \xi^{6}+63 \xi^{4}+37 \xi^{2}+11\right)(2 a m)^{6} \\
& +32\left(13 \xi^{12}+40 \xi^{10}+81 \xi^{8}+96 \xi^{6}+81 \xi^{4}+40 \xi^{2}+13\right)(2 a m)^{4} \\
& +64\left(\xi^{2}+1\right)\left(2 \xi^{12}+2 \xi^{10}+8 \xi^{8}+5 \xi^{6}+8 \xi^{4}+2 \xi^{2}+2\right)(2 a m)^{2}
\end{aligned}
$$

The chiral condensate, which is the derivative of $\log (Z(\xi, m))$ with respect to the bare fermion mass, $2 a m$, matches the data obtained using the mesonic worm algorithm (see Fig.3).

For $\beta>0$, the partition function is harder to compute exactly, since it involves the exact enumeration of self-avoiding loops on a periodic lattice. For $2 \times N_{t}$ lattices, however, the computation is feasible. For this reason, we test the full worm algorithm (mesonic + fermionic) on a $2 \times 2$ lattice, 


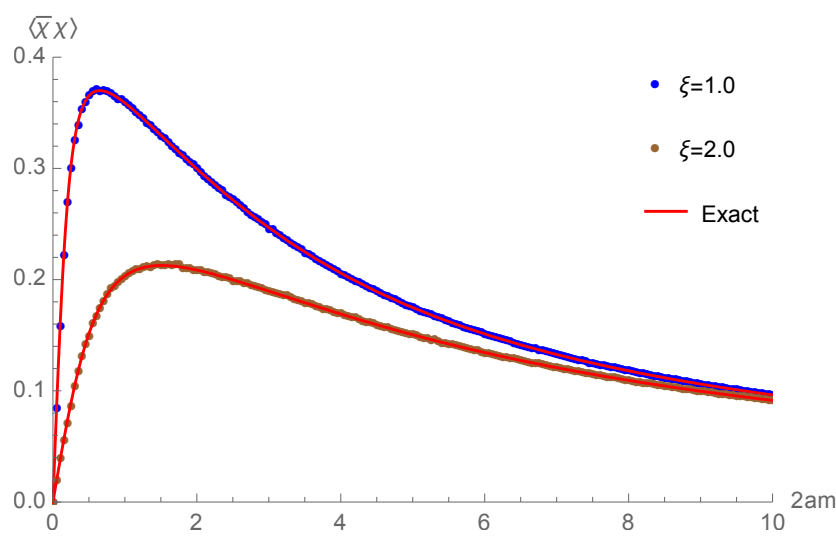

Figure 3: Chiral condensate at $\beta=0$ on a $4 \times 4$ lattice, using the mesonic worm algorithm.

whose partition function is given by (for $\xi=1$ ):

$$
Z(\beta, m)=\left((2 a m)^{4}+8(2 a m)^{2}+8\right) \sum_{n} I_{n}^{4}(\beta)+8 \sum_{n} I_{n}^{3}(\beta) I_{n+1}(\beta)+4 \sum_{n} I_{n}^{2}(\beta) I_{n+1}^{2}(\beta)
$$

where $I_{n}$ are modified Bessel functions of the first kind. The auxiliary variables are also updated. The chiral condensate as a function of $\beta$ and $2 \mathrm{am}$, almost matches the preliminary data from the simulations (see Fig.4).

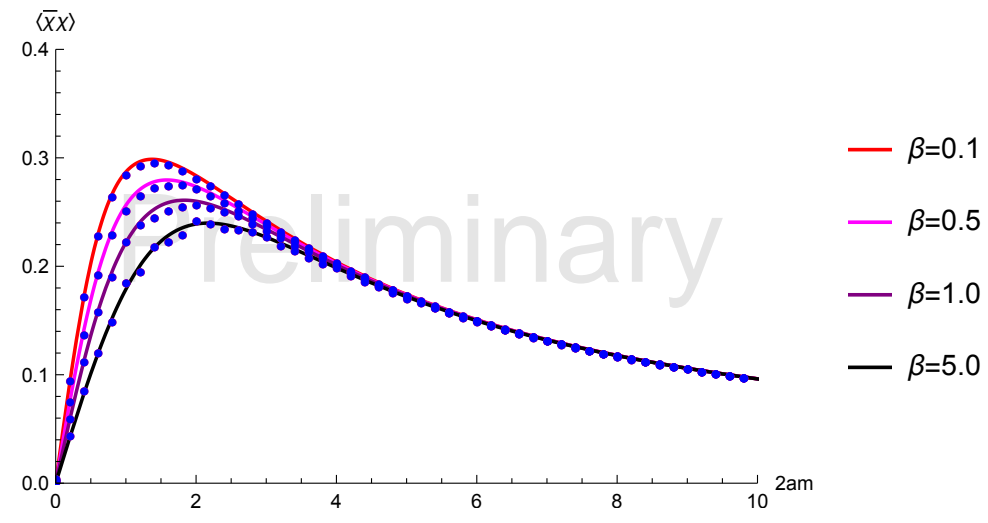

Figure 4: Chiral condensate for $\beta>0$ on a $2 \times 2$ lattice, using both the mesonic and the fermionic worm algorithms. The solid lines show the exact results from (3.2);

\section{Sign problem in the lattice Schwinger model}

For Monte Carlo simulations to be possible, we quench the total sign $\sigma$ of admissible configurations, and reweigh observables in the usual way: $\langle\mathscr{O}\rangle=\langle\sigma \mathscr{O}\rangle_{\|} /\langle\sigma\rangle_{\|}$. The total sign has two contributions, $\sigma=\sigma_{F} \sigma_{B}$ : a fermionic sign $\sigma_{F}$ from the geometry and topology of the fermion loops, and a bosonic sign $\sigma_{B}$ from fluctuations of the effective Wilson operators associated with fermion loops:

$$
\sigma_{B}(C)=\operatorname{sign}\left(\prod_{i=1}^{\# C} 2 \operatorname{Re}\left(\mathscr{U}\left(C_{i}\right)\right)\right)
$$


A useful quantity, which measures the severity of the sign problem in a lattice with volume $V$, is the difference $\Delta f$ in free energy density between the true and the quenched ensembles: $\sigma=e^{-V \Delta f}$. This quantity has a well-defined thermodynamic limit: it is zero when the two ensembles coincide, and grows as the sign problem becomes more severe. In the same spirit, we define similar quantities for the fermionic and bosonic signs, respectively $\sigma_{F}=e^{-V \Delta f_{F}}$ and $\sigma_{B}=e^{-V \Delta f_{B}}$.

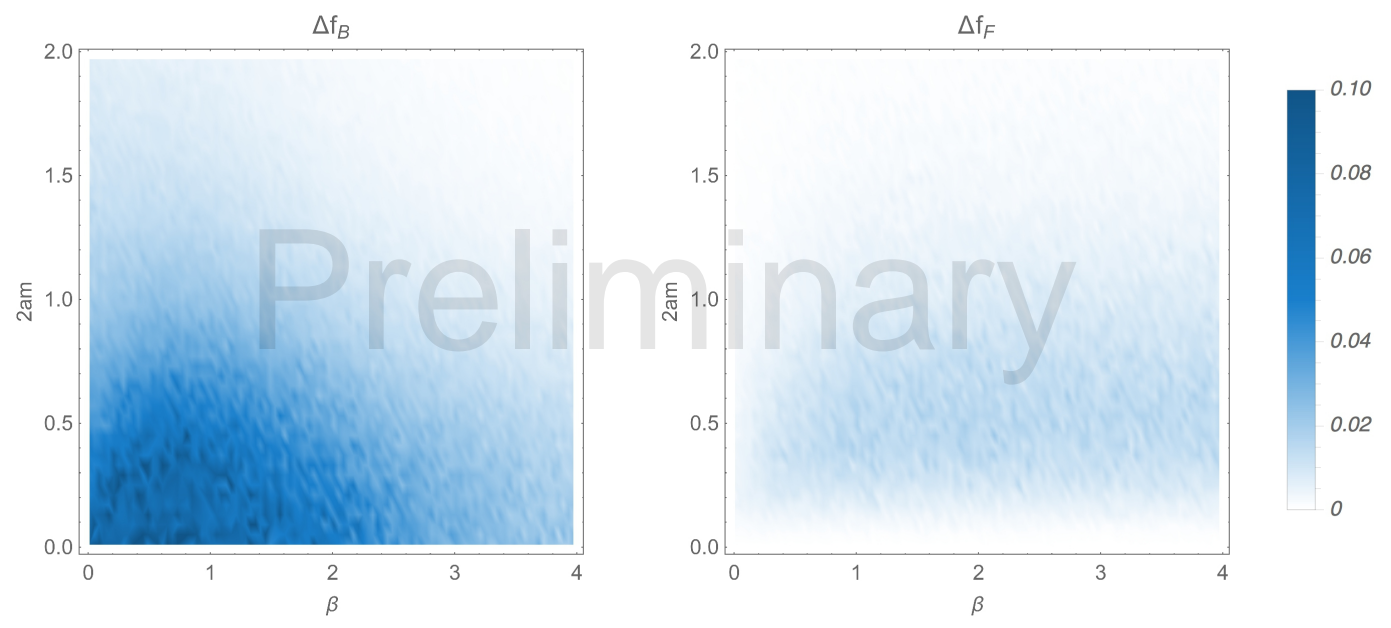

Figure 5: Severity of the sign problems, bosonic (left) and fermionic (right), in sign-quenched simulations of the massive lattice Schwinger model. Darker regions indicate a more severe sign problem.

In Fig.5 we show the density plots of $\Delta f_{B}$ and $\Delta f_{F}$ in a simulation of the massive Schwinger model on a $8 \times 8$ lattice. The fermionic sign problem is less mild within a range of small fermion masses, and approximately $\beta$-independent. For large masses, the lattice becomes saturated with monomers, leaving no room for fermion loops, thus resulting in the absence of a sign problem. In the chiral limit, however, the sign problem also disappears, despite the scarcity of monomers.

The bosonic sign problem, on the other hand, becomes more severe in the strong coupling limit, for small fermion masses. This is due to the fact that the positive expectation value of the distribution of effective Wilson loops is very close to zero at strong coupling, with a tail extending to negative values. This results in a significant fraction of the fermion loops generated in that region having a negative sign, which contributes to the suppression of $\left\langle\sigma_{B}\right\rangle$. At weak coupling, on the contrary, the distributions of Wilson loops are centered near 1, which implies that negative Wilson loops are highly suppressed, and consequently $\left\langle\sigma_{B}\right\rangle \approx 1$.

\section{Conclusion}

We showed that compact lattice QED with a single fermion flavour has an exact diagrammatic representation for arbitrary $\beta$, amenable to Monte Carlo simulations using worm-like algorithms. The method used to obtain this representation - the exact integration of the unitary link variables by coupling them to auxiliary bosonic fields living on plaquettes - can be extended straightforwardly to the non-Abelian and/or multi-flavour case, and provides a promising tool for simulating lattice gauge theories at finite density from first principles. For example, in the case of $N_{f}=1$ 
lattice $\mathrm{QCD}$, the admissible configurations at $\beta>0$ extend those at $\beta=0$ (i.e. configurations of monomers, dimers, and baryon loops) by simply adding a new ingredient: quark hoppings, whose weights depend on the effective Wilson loops along their worldlines (see Fig. 6), and which physically represent the microscopic/partonic structure of mesons and baryons, inaccessible at strong couplings.

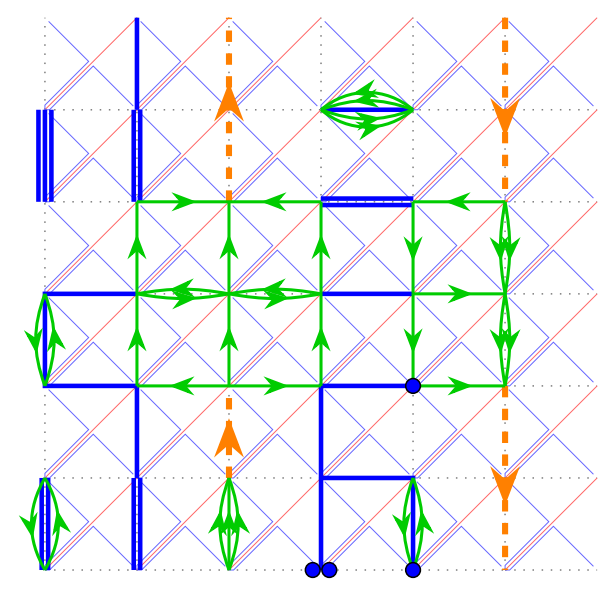

Figure 6: Admissible configuration of $N_{f}=1$ lattice QCD (in $2 d$ ) at finite $\beta$ : thick lines represent fermionic worldlines (blue for condensates and meson hoppings, orange for baryon hoppings, and green for quark hoppings), while the thin lines represent auxiliary bosonic variables. Functional Grassmann integration constrains the number of quarks to exactly 3 per site (and the same for antiquarks).

We use $N_{f}=1$ compact lattice QED as a toy model for this method. Despite of it lacking a chemical potential, due to Gauss' law, it can be used to benchmark the battery of numerical algorithms necessary to simulate similar systems (namely worm algorithms for the fermion worldlines, mesonic or fermionic, and a Gaussian heatbath for the auxiliary variables with Wilson loop corrections), and to study the effect of reweighing with respect to the sign-quenched partition function.

Preliminary simulations of the massive lattice Schwinger model show a severe sign problem in the strong coupling limit, due to the distributions of Wilson loops having a positive expectation value very close to zero, with a significant tail onto negative values. This obstacle limits the comparison with the diagrammatic methods used at strong coupling [4], which do not suffer from the bosonic sign problem observed here. The reason is that the (positive) coefficients of the bosonic weights can be computed analytically in a strong coupling expansion, but not for general $\beta$.

\section{References}

[1] P. de Forcrand, PoS LATT 2009 (2009) 010

[2] P. Rossi and U. Wolff, Nucl. Phys. B248 (1984) 105;

F. Karsch and K. H. Mutter, Nucl. Phys. B313 (1989) 541.

[3] N. Prokof'ev and B. Svistunov, Phys.Rev.Lett. 87 (2001) 160601,

D. H. Adams and S. Chandrasekharan, Nucl.Phys. B662 (2003) 220-246,

S. Chandrasekharan and F.-J. Jiang, Phys.Rev. D74 (2006) 014506,

[4] P. de Forcrand, J. Langelage, O. Philipsen and W. Unger, Phys. Rev. Lett. 113 (2014) 15, 152002

[5] H. Vairinhos and P. de Forcrand, JHEP 1412 (2014) 038 